\title{
UNIPOTENT CHARACTERS OF THE EVEN ORTHOGONAL GROUPS OVER A FINITE FIELD
}

\author{
BY
}

GEORGE LUSZTIG

\begin{abstract}
The characters of unipotent representations of a simple algebraic group over $F_{q}$ of type $\neq D_{n}$ on any regular semisimple element are explicitly known for large $q$. This paper deals with the remaining case: type $D_{n}$.
\end{abstract}

The purpose of this paper is to give explicit formulas for the character of the unipotent representations of the special orthogonal group of a quadratic form of even dimension over a finite field $F_{q}$ on any regular semisimple element, provided that $q$ is sufficiently large. The methods used in this paper are those of [4], where the case of symplectic and odd orthogonal groups was considered. To avoid repetitions, I have only given proofs for the results which differ essentially from those in [4].

1. Characters of Weyl groups and Hecke algebras of type $D_{n}$.

1.1. Let $D_{n}(n \geqslant 2)$ be the Coxeter group with diagram

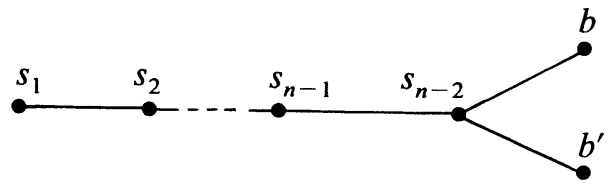

(Here $s_{1}, s_{2}, \ldots, s_{n-2}, b, b^{\prime}$ are the simple reflections.) For $n=2$, this is the group generated by the commuting involutions $b, b^{\prime}$. We make the convention that $D_{1}$ is the group with 1 element. We may regard $D_{n}(n \geqslant 1)$ as a subgroup of index 2 of the group $\hat{D}_{n}$ generated by $D_{n}$ and an element $\varphi$ of order 2 which commutes with all $s_{i}$ $(1 \leqslant i \leqslant n-2)$ and satisfies $\varphi b \varphi=b^{\prime}$ (if $n \geqslant 2$ ).

1.2. The group $\hat{D}_{n}$ is isomorphic in two ways with the Coxeter group of type $B_{n}$ (denoted $W_{n}$ in [4]): One choice of simple reflections is $\left\{s_{1}, s_{2}, \ldots, s_{n-2}, b, \varphi\right\}$; the other is $\left\{s_{1}, s_{2}, \ldots, s_{n-2}, b^{\prime}, \varphi\right\}$. Each of these two choices gives rise to an isomorphism $\hat{D}_{n} \approx W_{n}$ of Coxeter systems. We may thus parametrize the irreducible $\mathbf{Q}\left[\hat{D}_{n}\right]$-modules by ordered pairs $\sigma_{1}, \sigma_{2}$ of irreducible representations of the symmetric groups $\widetilde{S}_{k}, S_{l}(k+l=n)$, as in $[4,2.1]$ and this parametrization will be independent of the choice of simple reflections for $\hat{D}_{n}$ (the two choices are conjugate by $\varphi$ ). It will be convenient to parametrize the irreducible $Q\left[\hat{D}_{n}\right]$-modules by ordered pairs $(\underset{T}{S})$ where $S, T$ are finite subsets of $\{0,1,2, \ldots\}$ defined as follows: if $\sigma_{1}, \sigma_{2}$

Received by the editors May 19, 1981.

1980 Mathematics Subject Classification. Primary 20G40.

'Supported in part by the National Science Foundation. 
correspond to the partitions $0 \leqslant \alpha_{1} \leqslant \alpha_{2} \leqslant \cdots \leqslant \alpha_{m}, 0 \leqslant \beta_{1} \leqslant \beta_{2} \leqslant \cdots \leqslant \beta_{m}$ of $k$, $l$ respectively, we set $S=\left\{\alpha_{1}, \alpha_{2}+1, \ldots, \alpha_{m}+m-1\right\}, T=\left\{\beta_{1}, \beta_{2}+1, \ldots, \beta_{m}+\right.$ $m-1$ \}. Here, $m$ can be increased at our will, so that $\left({ }_{T}^{S}\right)$ should be regarded as being equivalent to

$$
\left(\begin{array}{l}
0 \cup(S+1) \\
0 \cup(T+1)
\end{array}\right)
$$

and also to pairs obtained from $(\underset{T}{S})$ by iterating this "shift" operation. Thus, we have a 1-1 correspondence

$$
\left(\begin{array}{l}
S \\
T
\end{array}\right) \leftrightarrow\left[\begin{array}{l}
S \\
T
\end{array}\right]
$$

between the set

$F_{n}=\left\{\left(\begin{array}{l}S \\ T\end{array}\right) \mid\right.$ ordered pair with $S, T \subset\{0,1,2, \ldots\},|S|=|T|=m$,

$$
\left.\sum_{\lambda_{i} \in S} \lambda_{i}+\sum_{\mu_{i} \in T} \mu_{i}=n+m^{2}-m, \text { up to shift }\right\}
$$

and the set of irreducible $\mathbf{Q}\left[\hat{D}_{n}\right]$-modules (up to isomorphism). Here $\left[\begin{array}{l}S \\ T\end{array}\right]$ is the $\mathbf{Q}\left[\hat{D}_{n}\right]$-module corresponding to $\left({ }_{T}^{S}\right)$. This parametrization is more appropriate for the study of groups of type $D_{n}$ than that in $[4,2.1]$. It follows from the definition that

$$
\left[\begin{array}{l}
S \\
T
\end{array}\right] \otimes \chi=\left[\begin{array}{l}
T \\
S
\end{array}\right]
$$

where $\chi: \hat{D}_{n} \rightarrow\{ \pm 1\}$ is the homomorphism whose kernel is $D_{n}$.

1.3. Let $w_{0}$ be the longest element of the Coxeter group $D_{n}$. Then $\varphi^{n} w_{0}$ is the unique nontrivial central element of $\hat{D}_{n}$. It acts on the irreducible $\mathbf{Q}\left[\hat{D}_{n}\right]$-module $\left[{ }_{T}^{S}\right]$ as multiplication by the scalar

$$
(-1)^{\sum_{i=1}^{m}\left(\mu_{i}-i+1\right)}
$$

where $T=\left\{\mu_{1}, \mu_{2}, \ldots, \mu_{m}\right\}$. This follows immediately from [4, 2.2].

1.4. Let $\operatorname{sign}^{\prime}$ be the unique homomorphism $\hat{D}_{n} \rightarrow\{ \pm 1\}$ such that $\operatorname{sign}^{\prime}(\varphi)=1$ and sign' restricted to $D_{n}$ is the sign character of the Coxeter group $D_{n}$. Let $\left(\begin{array}{c}S \\ T\end{array}\right) \in F_{n}$; assume that it is represented by sets $S, T$ with $|S|=|T|=m$. Choose an integer $t$ which is greater than or equal to any entry of $S$ and $T$. Let $\bar{S}=\{t-i \mid 0 \leqslant i \leqslant t$, $i \notin S\}, \bar{T}=\{t-i \mid 0 \leqslant i \leqslant t, i \notin T\}$. Then $\left(\frac{\bar{S}}{T}\right)$ is independent of the choice of $t$ (up to shift); it defines an element of $F_{n}$, and we have

$$
\left[\begin{array}{l}
S \\
T
\end{array}\right] \otimes \operatorname{sign}^{\prime}=\left[\begin{array}{l}
\bar{S} \\
\bar{T}
\end{array}\right]
$$

as representations of $\hat{D}_{n}$. This follows from $[4,2.4]$ together with (1.2.1).

1.5. Let $H$ be the Hecke algebra (with coefficients in $\mathrm{Q}\left[u^{1 / 2}, u^{-1 / 2}\right]$ ) corresponding to the Coxeter group $D_{n}$; here $u^{1 / 2}$ is an indeterminate. Let $\hat{H}$ be the algebra over $\mathbf{Q}\left[u^{1 / 2}, u^{-1 / 2}\right]$ generated by $H$ and by an element $T_{\varphi}$ with the relations $T_{\varphi}^{2}=1$, $T_{\varphi} T_{w} T_{\varphi}=T_{\varphi w \varphi}\left(w \in D_{n}\right)$, where $T_{w}\left(w \in D_{n}\right)$ are the canonical basis elements of $H$. 
Thus, $H$ is a subalgebra of $\hat{H}$ which, under the specialization $u^{1 / 2} \rightarrow 1$, becomes the subalgebra $\mathbf{Q}\left[D_{n}\right]$ of $\mathbf{Q}\left[\hat{D}_{n}\right]$.

Let $E$ be an irreducible $\mathrm{Q}\left[\hat{D}_{n}\right]$-module whose restriction $E \mid D_{n}$ to $D_{n}$ is irreducible. The construction in $[4,1.1]$ with $W=D_{n}$ associates to $E \mid D_{n}$ an irreducible $H$-module $\tilde{E}=\left(M_{C} \otimes_{\mathrm{Q}} E\right)^{D_{n}}$ (where $C \subset D_{n}$ is the two-sided cell of $D_{n}$ corresponding to $\left.E \mid D_{n}\right)$. Note that $\tilde{E}$ is free as a $\mathbf{Q}\left[u^{1 / 2}, u^{-1 / 2}\right]$-module. It has a natural involution which takes an element $\Sigma_{z \in C} e_{z} \otimes v_{z}$ to $\Sigma_{z \in C} e_{\varphi z \varphi} \otimes \varphi\left(v_{z}\right)$. (Here $e_{z}, z \in C$, are the basis elements of $M_{C}$, see $[4,1.1]$.) This involution of $\tilde{E}$ is denoted $T_{\varphi}$. Together with the $H$-module structure on $\tilde{E}$, it defines an $\hat{H}$-module structure on $\tilde{E}$.

We have, as in $[4,1.2]$,

$$
\operatorname{Tr}\left(T_{x} T_{\varphi}^{i}, \tilde{E}\right) \in \mathbf{Z}\left[u^{1 / 2}\right] \quad\left(i=0,1 ; x \in D_{n}\right) .
$$

Moreover, with the notations in $[4,1.2]$ we have

$$
\begin{array}{r}
\operatorname{Tr}\left(T_{x} T_{\varphi}^{i}, \tilde{E}\right)=\left.\left|D_{n}\right|^{-1} \sum_{w \in D_{n}} \sum_{\substack{z, z^{\prime} \in C \\
\varphi^{i} z \varphi_{\tilde{L}^{\prime}}^{\prime} z^{\prime} \tilde{R}^{z}}} \tau\left(T_{x} C_{\varphi^{i} z \varphi^{i}} D_{z^{\prime-1}}\right) \tau\left(D_{z^{-1}} C_{z^{\prime}} T_{w}\right)\right|_{u=1} \operatorname{Tr}\left(w \varphi^{i}, E\right) \\
\left(i=0,1 ; x \in D_{n}\right) .
\end{array}
$$

The proof is the same as that of $[4,1.3]$. This implies, as in $[4,1.6]$, that

$$
\operatorname{Tr}\left(T_{x^{-1}}^{-1} T_{\varphi}^{i}, \tilde{E}\right)=\overline{\operatorname{Tr}\left(T_{x} T_{\varphi}^{i}, \tilde{E}\right)} \quad\left(i=0,1 ; x \in D_{n}\right),
$$

where $a \rightarrow \bar{a}$ is the involution of the ring $\mathbf{Q}\left[u^{1 / 2}, u^{-1 / 2}\right]$ such that $\overline{u^{j / 2}}=u^{-j / 2}$. The following result is proved in the same way as $[4,1.7]$ :

$$
\operatorname{Tr}\left(T_{x} T_{\varphi}^{i}, \tilde{E}\right)=\operatorname{Tr}\left(T_{x^{-1}} T_{\varphi}^{i}, \tilde{E}\right) \quad\left(i=0,1 ; x \in D_{n}\right) .
$$

Let $\operatorname{Dim}(\tilde{E})$ be the "formal dimension" of the $H$-module $\tilde{E}$ (an element of $\mathbf{Q}[u]$ ). We have the identity $(i=0$ or 1$)$ :

$$
\sum_{x \in D_{n}} u^{-l(x)} \operatorname{Tr}\left(T_{x} T_{\varphi}^{i}, \tilde{E}\right) \operatorname{Tr}\left(T_{x^{-1}} T_{\varphi}^{i}, \tilde{E}\right)=\frac{\sum_{w \in D_{n}} u^{l(w)}}{\operatorname{Dim}(\tilde{E})} \cdot \operatorname{dim}(E)
$$

(where $l$ is the length function on $D_{n}$ ).

For $i=0$, this is a special case of $[4,(1.8 .1)]$. We now prove it for $i=1$. The left-hand side of (1.5.5) is the trace of the $\mathbf{Q}\left(u^{1 / 2}\right)$-linear map

$$
h \rightarrow \sum_{x \in D_{n}} u^{-l(x)} T_{x} T_{\varphi}^{i} h T_{\varphi}^{i} T_{x^{-1}}: J \rightarrow J
$$

where $J$ is the minimal 2-sided ideal of $H \otimes \mathbf{Q}\left(u^{1 / 2}\right)$ corresponding to the irreducible representation $\tilde{E} \otimes \mathbf{Q}\left(u^{1 / 2}\right)$. But one checks easily that $\Sigma_{x \in D_{n}} u^{-l(x)} T_{x} h^{\prime} T_{x^{-1}}$ is in the centre of $H \otimes \mathbf{Q}\left(u^{1 / 2}\right)$ for all $h^{\prime} \in H \otimes \mathbf{Q}\left(u^{1 / 2}\right)$. Hence our linear map has image consisting of scalar multiples of a single vector $v$, which spans the intersection of $J$ with the centre. Hence its trace is equal to $\lambda$ where $\lambda v=$ $\sum_{x \in D_{n}} u^{-l(x)} T_{x} T_{\varphi}^{i} v T_{\varphi}^{i} T_{x^{-1}}$. But $v$ must be also in the centre of $\hat{H}$ (since $\tilde{E}$ is an $\hat{H}$-module); thus $T_{\varphi}^{i} v T_{\varphi}^{i}=v$ and so $\lambda$ is independent of $i$, as required. 
Let us define integers $A(E), a(E)$ (cf. $[4,1.8])$ as follows: $A(E)$ is the degree of the polynomial (in $u) \operatorname{Dim}(\tilde{E})$ and $u^{a(E)}$ is the largest power of $u$ dividing this polynomial. (These should not be confused with the integers $A(E), a(E)$ defined using a Coxeter group structure of type $B_{n}$ on $\hat{D}_{n}$, which are different.) Using (1.5.4), (1.5.5), as in [4, 1.9], we deduce that for $i=0$ and 1 , and $x \in D_{n}$, we have

$$
\operatorname{Tr}\left(T_{x} T_{\varphi}^{i}, \tilde{E}\right)=\left\{\begin{array}{l}
\text { constant } \cdot u^{(l(x)-a(E)) / 2}+\text { higher powers of } u^{1 / 2} \\
\text { constant } \cdot u^{(l(x)-A(E)+\nu) / 2}+\text { lower powers of } u^{1 / 2}
\end{array}\right.
$$

(Here $\nu=n^{2}-n$ is the length of $w_{0} \in D_{n}$.) As in $[4,1.11]$ we deduce that

$$
\begin{aligned}
& T_{w_{0}} T_{\varphi}^{n} \text { acts on } \tilde{E} \text { as scalar multiplication by } \pm u^{\nu-(a(E)+A(E)) / 2} \text { and } \\
& \operatorname{Tr}\left(T_{x w_{0}} T_{\varphi}^{i}, \tilde{E}\right)= \pm \overline{\operatorname{Tr}\left(T_{x} T_{\varphi}^{i+n}, \tilde{E}\right)}
\end{aligned}
$$

where the sign \pm 1 is the scalar by which $w_{0} \varphi^{n}$ acts on $E\left(i=0,1 ; x \in D_{n}\right)$. We have (1.5.8) $\operatorname{Tr}\left(T_{x} T_{\varphi}^{i},\left(E \otimes \operatorname{sign}^{\prime}\right)^{\tilde{y}}\right)=(-u)^{l(x)} \operatorname{Tr}\left(T_{x} T_{\varphi}^{i}, \tilde{E}\right) \quad\left(i=0,1 ; x \in D_{n}\right)$

where sign' is defined as in 1.4. (Compare $[4,1.13]$.) We now define

$$
\operatorname{Tr}\left(T_{x} T_{\varphi}^{i}, \tilde{E} ; h / 2\right) \in \mathbf{Z} \quad\left(i=0,1 ; x \in D_{n}\right)
$$

to be the coefficient of $u^{h / 2}$ in $\operatorname{Tr}\left(T_{x} T_{\varphi}^{i}, \tilde{E}\right)$, see (1.5.1). For each $x \in D_{n}$, we define $\alpha_{x}, \mathscr{Q}_{x} \in \mathcal{R}\left(D_{n}\right)$ (= group of virtual representations of $D_{n}$ ) by the formulas [4, (1.15.1), (1.15.2)], with $W=D_{n}$. We also define two virtual $\hat{D}_{n}$-representations $\alpha_{x \varphi}$, $\mathbb{Q}_{x \varphi}$ by the formulas:

$$
\begin{gathered}
\alpha_{x \varphi}=(-1)^{l(x)} \sum_{E} \operatorname{Tr}\left(T_{x} T_{\varphi}, \tilde{E} ; \frac{l(x)-a(E)}{2}\right) E ; \\
\mathcal{Q}_{x \varphi}=\sum_{E} \operatorname{Tr}\left(T_{x} T_{\varphi} \tilde{E} ; \frac{l(x)-A(E)+\nu}{2}\right) E ;
\end{gathered}
$$

both sums are over all irreducible $\hat{D}_{n}$-modules $E$ which remain irreducible on restriction to $D_{n}, l(x)$ is the length in the Coxeter group $D_{n}$ and $\nu=n^{2}-n$. Thus, $\alpha_{x \varphi}$ and $A_{x \varphi}$ belong to $\Re\left(\hat{D}_{n}\right)^{-}$, the group of virtual representations of $\hat{D}_{n}$ which are anti-invariant under the $Z$-linear involution $\iota$ which interchanges any two distinct irreducible representations of $\hat{D}_{n}$ which are the same as $D_{n}$-modules, and keeps fixed any irreducible representation of $\hat{D}_{n}$ which is reducible as a $D_{n}$-module. We have (cf. $(1.5 .8),[4,1.14,1.16])$

$$
\mathbb{Q}_{x}=\alpha_{x} \otimes\left(\operatorname{sign}^{\prime} \mid D_{n}\right), \quad \mathbb{Q}_{x \varphi}=\alpha_{x \varphi} \otimes \operatorname{sign}^{\prime} .
$$

In the case where $n$ is even, so that $w_{0}$ is in the centre of $D_{n}$ of $\hat{D}_{n}$, we have defined in $[4,1.17]$ a $Z$-linear involution $\zeta$ of the group $\Re\left(D_{n}\right)$ : it sends an irreducible $D_{n}$-module $E$ to $\pm E$ where \pm 1 is the sign by which $w_{0}$ acts on $E$. We can also define a Z-linear involution of the group of virtual representations of $\hat{D}_{n}$ which sends an irreducible $\hat{D}_{n}$-module $E$ to $\pm E$ where \pm 1 is the sign by which $w_{0}$ acts on $E$; we denote again by $\zeta$, the restriction of this involution to the group $\Re\left(\hat{D}_{n}\right)^{-}$. (It is mapped into itself by $\zeta$.) 
In the case where $n$ is odd, so that $w_{0} \varphi$ is in the centre of $\hat{D}_{n}$ we define two $\mathbf{Z}$-linear bijections (inverse to each other)

$$
\Re\left(D_{n}\right) \underset{\zeta}{\stackrel{\zeta}{\rightleftarrows}} \Re\left(\hat{D}_{n}\right)^{-}
$$

as follows. If $E$ is an irreducible $D_{n}$-module, let $E^{\prime}$ (resp. $E^{\prime \prime}$ ) be the unique $\hat{D}_{n}$-module which becomes $E$ on restriction to $D_{n}$ and is such that $w_{0} \varphi$ acts on it as +1 (resp. as -1$)$. We set $\zeta(E)=E^{\prime}-E^{\prime \prime}$. This gives one of the bijections $\zeta$. The other one is its inverse.

We have

$$
\mathbb{Q}_{x \varphi^{i} w_{0}}=(-1)^{l(x)} \zeta\left(\alpha_{x \varphi^{i+n}}\right) \quad\left(i=0,1 ; x \in D_{n}\right)
$$

where $\zeta$ has four possible meanings (explained above) according to whether $i$ and $n$ are even or odd.

1.6. The irreducible $\mathbf{Q}\left[D_{n}\right]$-modules can be parametrized as follows. If $E$ is such a module and if $E$ can be extended to a $\hat{D}_{n}$-module $\left[{ }_{T}^{S}\right]$, then we associate to $E$ the unordered pair $\left({ }_{T}^{S}\right)$, and we still denote $E=\left[{ }_{T}^{S}\right]$; note that the other $\hat{D}_{n}$-module extending $E$ is $\left[{ }_{S}^{T}\right]$ (cf. (1.2.1)), so the unordered pair $S, T$ is well-defined (up to shift); it is a symbol of rank $n$ and defect 0 (up to shift) (see [2, §3]). If $E$ cannot be extended to a $\hat{D}_{n}$-module, then the representation induced by $E$ to $\hat{D}_{n}$ is irreducible, of form $\left[\begin{array}{l}S \\ S\end{array}\right]$. We associate to $E$ the symbol $\left(\begin{array}{c}S \\ S\end{array}\right)$, up to shift; in this case, the symbol does not determine the isomorphism class of $E$ uniquely: there are two irreducible representations of $D_{n}$ corresponding to it. Thus we have a correspondence

$$
\left[\begin{array}{l}
S \\
T
\end{array}\right] \rightarrow\left(\begin{array}{l}
S \\
T
\end{array}\right)
$$

between irreducible representations of $D_{n}$ and symbols of rank $n$ and defect 0 (up to shift) which is 1-1 except that to symbols of form $(\underset{S}{S})$ (said to be degenerate) correspond two representations of $D_{n}$ (also said to be degenerate).

According to $[4,1.8]$, there are well-defined invariants $a(E), A(E), \gamma_{E}$ of an irreducible $D_{n}$-module $E$. (When $E$ extends to an irreducible representation $E^{\prime}$ of $\hat{D}_{n}$, then $a(E), A(E)$ are the same as $a\left(E^{\prime}\right), A\left(E^{\prime}\right)$, as defined in 1.5.) If $E$ corresponds to

$$
\left(\begin{array}{l}
S \\
T
\end{array}\right)=\left(\begin{array}{l}
\lambda_{1}, \ldots, \lambda_{m} \\
\mu_{1}, \ldots, \mu_{m}
\end{array}\right)
$$

we have

$$
\begin{gathered}
a(E)=\sum_{i<j} \inf \left(\lambda_{i}, \lambda_{j}\right)+\sum_{i<j} \inf \left(\mu_{i}, \mu_{j}\right) \\
+\sum_{i, j} \inf \left(\lambda_{i}, \mu_{j}\right)-\frac{m}{6}(m-1)(4 m-5), \\
\gamma_{E}= \begin{cases}2^{-(d-1)}, & \text { if } d \geqslant 1, \\
1, & \text { if } d=0,\end{cases}
\end{gathered}
$$

where $2 d=(S \cup T)-(S \cap T)$ is the number of "singles" in $(\underset{T}{S})$. 
1.7. The proofs of the statements in this section are similar to those of $[4,2.7,2.8]$. Assume that $n \geqslant 2$ and that $r+s=n$ is a partition of $n$ such that $n \geqslant 1, s \geqslant 1$. We identify $\varsigma_{r} \times \hat{D_{s}}$ with the subgroup of $\hat{D_{n}}$ generated by $s_{1}, \ldots, s_{r-1}, s_{r+1}, \ldots, s_{n-2}, b, b^{\prime}, \varphi$ and $\varsigma_{r} \times D_{s}$ with the corresponding subgroup of $D_{n}$. (Thus $\Im_{r} \times D_{s}$ is the intersection of $\Im_{r} \times \hat{D}_{s}$ with $D_{n}$.)

Let $\varepsilon(r)$ be the sign representation of $\Im_{r}$. We wish to describe the $D_{n}$-module $J_{\mathscr{S}_{r}, \times D_{s}}^{D_{n}}\left(\varepsilon(r) \otimes\left[{ }_{T}^{S}\right]\right)$ (see $\left.[4,1.19]\right)$ where $\left[{ }_{T}^{S}\right]$ is an irreducible representation of $D_{s}$ with $S \neq T$. We may assume that $2 m \geqslant r$. This $D_{n}$-module is $\left[\begin{array}{c}S^{\prime} \\ T^{\prime}\end{array}\right]$ or $\left[\begin{array}{c}S^{\prime} \\ T^{\prime}\end{array}\right]+\left[\begin{array}{l}S^{\prime \prime} \\ T^{\prime \prime}\end{array}\right]$, where $\left(\begin{array}{c}S^{\prime} \\ S^{\prime}\end{array}\right),\left(\begin{array}{c}S^{\prime \prime} \\ T^{\prime \prime}\end{array}\right)$ are obtained from $\left(\begin{array}{c}S \\ T\end{array}\right)$ by increasing each of the $n$ largest entries in $S$, $T$ by 1 and leaving the others unchanged. This process can be done either in a unique way or in two distinct ways (see [4, 2.6]) and it leads either to a unique symbol $\left(\begin{array}{c}S^{\prime} \\ T^{\prime}\end{array}\right)$ with $S^{\prime} \neq T^{\prime}$ or to two symbols $\left(\begin{array}{c}S^{\prime} \\ T^{\prime}\end{array}\right),\left(\begin{array}{c}S^{\prime \prime} \\ T^{\prime \prime}\end{array}\right)$ with $S^{\prime} \neq T^{\prime}, S^{\prime \prime} \neq T^{\prime \prime}$. In the case where $S=T$, the same process leads again either to a unique symbol $\left(\begin{array}{c}S^{\prime} \\ T^{\prime}\end{array}\right)$ with $S^{\prime}=T^{\prime}$ (if $r$ is even) or to two symbols $\left(\begin{array}{c}S^{\prime} \\ T^{\prime}\end{array}\right),\left(\begin{array}{c}S^{\prime \prime} \\ T^{\prime \prime}\end{array}\right)$ with $S^{\prime} \neq T^{\prime}, S^{\prime \prime} \neq T^{\prime \prime}, S^{\prime}=T^{\prime \prime}$, $S^{\prime \prime}=T^{\prime}$ (if $r$ is odd); we have

$$
J_{\Xi_{r} \times D_{s}}^{D_{n}}\left(\varepsilon(r) \otimes\left[\begin{array}{l}
S \\
T
\end{array}\right]\right)=\left[\begin{array}{l}
S^{\prime} \\
T^{\prime}
\end{array}\right] .
$$

Here $\left[{ }_{T}^{S}\right]$ can have 2 possible meanings (since $S=T$ ), but if $r$ is odd, the formula holds with both these meanings: $\left[{ }_{T^{\prime}}^{S^{\prime}}\right]$ is well defined. If $r$ is even, $\left[\begin{array}{l}S \\ T\end{array}\right]$ and $\left[{ }_{T^{\prime}}^{S^{\prime}}\right]$ both have 2 possible meanings, and our formula is ambiguous. We will make it unambiguous as follows. One can label the two irreducible $D_{n}$-modules corresponding to a symbol $\left(\begin{array}{c}S^{\prime} \\ T^{\prime}\end{array}\right)$ with $S^{\prime}=T^{\prime}$ as $\left[{ }_{T^{\prime}}^{S^{\prime}}\right]_{b},\left[{ }_{T^{\prime}}^{S^{\prime}}\right]_{b^{\prime}}$ in such a way that (1.7.1) with $r, s, n$ even can be written in the unambiguous form:

$$
\left\{\begin{array}{l}
J_{\mathscr{S}_{r} \times D_{s}}^{D_{n}}\left(\varepsilon(r) \otimes\left[\begin{array}{l}
S \\
T
\end{array}\right]_{b}\right)=\left[\begin{array}{l}
S^{\prime} \\
T^{\prime}
\end{array}\right]_{b}, \\
J_{\mathscr{S}_{r} \times D_{s}}^{D_{n}}\left(\varepsilon(r) \otimes\left[\begin{array}{l}
S \\
T
\end{array}\right]_{b^{\prime}}\right)=\left[\begin{array}{l}
S^{\prime} \\
T^{\prime}
\end{array}\right]_{b^{\prime}} .
\end{array}\right.
$$

(Note that $b, b^{\prime}$ in $D_{n}$ and $b, b^{\prime}$ in $D_{s}$ are in natural 1-1 correspondence, $n \geqslant s \geqslant 2$.)

This labelling is unique if we require that it also has the following property. Let $\Im_{n}^{(b)}$ be the subgroup of $D_{n}(n \geqslant 2)$ generated by all simple reflections $\neq b^{\prime}$. (It is a symmetric group.) Similarly, we define $\Im_{n}^{\left(b^{\prime}\right)}=\varphi \varsigma_{n}^{(b)} \varphi$. Then

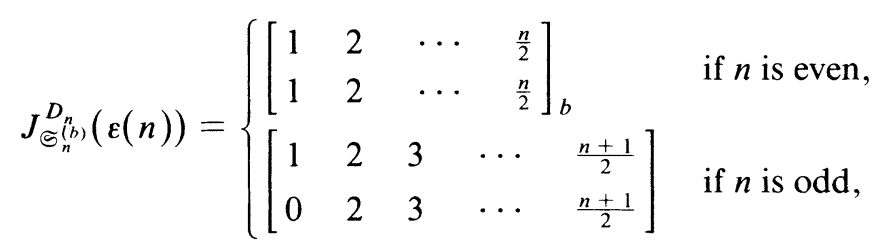

and the same holds with $b$ replaced by $b^{\prime}$. Note also that

$$
J_{\subseteq_{r} \times D_{s}}^{D_{n}}\left(\varepsilon(r) \otimes \alpha_{z}^{\left(D_{s}\right)}\right)=\alpha_{\left.w_{0}^{\prime}\right)_{z}}^{\left(D_{D^{\prime}}\right)} \quad(n=r+s, r \geqslant 1, s \geqslant 1)
$$

where $w_{0}^{(r)}$ is the longest element in $\subseteq_{r} ; z \in D_{s}, \alpha_{z}^{\left(D_{s}\right)}$ means $\alpha_{z}$ with respect to $D_{s}$; and $\alpha_{w_{0}^{(r) z}}^{\left(D_{n}\right)}$ means $\alpha_{w_{0}^{(r)} z}$ with respect to $D_{n}$; see [4, 1.20, 1.21, 1.22]. Moreover,

$$
J_{\Theta_{n}^{(b)}}^{D_{n}}(\varepsilon(n))=\alpha_{w_{0, b}^{n, ~}}^{\left(D_{n}\right)},
$$


where $w_{0, b}^{(n)}$ is the longest element in $\mathfrak{S}_{n}^{(b)}$; the same holds with $b$ replaced by $b^{\prime}$.

1.8. Assume that $n \geqslant 2$ and that $r+s=n$ is a partition of $n$ such that $r \geqslant 1$, $s \geqslant 1$. Consider an irreducible representation $\left[{ }_{T}^{S}\right]$ of $\hat{D}_{s}$ with $S \neq T(S, T$ is an ordered pair). We consider the $\hat{D}_{n}$-module $J_{\mathbb{S}_{r} \times D_{s}}^{D_{n}}\left(\varepsilon(r) \otimes\left[{ }_{T}^{S}\right]\right)$, defined in the same way as in [4, 1.19] except that the invariant $a(E)$ is defined as in 1.5 (using the Coxeter group structure of $D_{s}, D_{n}$ ). This $\hat{D}_{n}$-module is again $\left[\begin{array}{c}S_{T^{\prime}}^{\prime} \\ ]\end{array}\right]$ or $\left[\begin{array}{c}S^{\prime} \\ T^{\prime}\end{array}\right]+\left[\begin{array}{l}S_{T^{\prime \prime}}^{S^{\prime \prime}} \\ \text {, where }\end{array}\right.$ these are obtained from $S, T$ by increasing each of the $r$ largest entries in $S, T$ by 1 and leaving the others unchanged; we assume here, as we may, that $|S|=|T| \geqslant r$. (Note that $S^{\prime}, T^{\prime}$ and $S^{\prime \prime}, T^{\prime \prime}$ are ordered pairs.) The map $J_{\mathscr{S}_{r}, \times D_{s}}^{D_{n}}$ extends by $\mathbf{Z}$-linearity to a map

$$
\Re\left(\hat{D}_{s}\right)^{-} \rightarrow \Re\left(\hat{D}_{n}\right)^{-}
$$

We have

$$
J_{\mathbb{S}_{n}, \times \hat{D}_{s}}^{\hat{D}_{n}}\left(\alpha_{z \varphi}^{\left(\hat{D}_{s}\right)}\right)=\alpha_{w_{0}^{\left(r^{\prime}\right)} z \varphi}^{\left(\hat{D}_{n}\right)} \quad\left(z \in D_{s}\right)
$$

where the elements $\varphi$ of $\hat{D}_{s}, \hat{D}_{n}$ are identified via the natural inclusion $\hat{D}_{s} \subset \hat{D}_{n}$, and the upper-scripts $\left(\hat{D}_{s}\right),\left(\hat{D}_{n}\right)$ have a meaning similar to that in 1.7 .

1.9. Let

$$
Z=\left(\begin{array}{c}
z_{2}, z_{4}, \ldots, z_{2 m} \\
z_{1}, z_{3}, \ldots, z_{2 m-1}
\end{array}\right)
$$

be a tableau consisting of integers $z_{i}$ satisfying $z_{1}<z_{3}<\cdots<z_{2 m-1}, z_{2}<z_{4}<$ $\cdots<z_{2 m}, 0 \leqslant z_{1} \leqslant z_{2} \leqslant z_{3} \leqslant \cdots \leqslant z_{2 m-1} \leqslant z_{2 m}, \quad \sum_{i=1}^{2 m} z_{i}=n+m^{2}-m(n \geqslant 1)$. We say that $Z$ is degenerate if $z_{1}=z_{2}, z_{3}=z_{4}, \ldots, z_{2 m-1}=z_{2 m}$; otherwise, it is nondegenerate. We shall regard $Z$ as being equivalent to its shifts (see $\$ 1.2$ ). We say that $Z$ is a special symbol of rank $n$ and defect 0 . If $Z$ is nondegenerate, we denote by $[Z]$ the corresponding irreducible representation $\hat{D}_{n}$ (see $\S 1.2$ ). From (1.6.1) we see that $a[Z] \equiv \sum_{i=1}^{m}\left(z_{2 i-1}-i+1\right)(\bmod 2)$. Using 1.3 , it follows that $w_{0} \varphi^{n}$ acts on $[Z]$ as multiplication by $(-1)^{a[Z]}$.

1.10. Assume that $Z$ is nondegenerate. Then it has $2 d>0$ "singles" (= entries appearing in exactly one row of $Z$ ). Let $\Phi$ be an arrangement of the $2 d$ "singles" in $Z$ into $d$ disjoint pairs such that each pair in $\Phi$ contains one single in the first row of $Z$ and one in the second row of $Z$. We define what it means for $\Phi$ to be an admissible arrangement for $Z$ in the same way as in [4, 2.11], by induction on $d \geqslant 1$. (Compare [5].) Here we must start the induction with the case $d=1$ in which case the unique arrangement is, by definition, admissible. For example, $Z=\left(\begin{array}{ll}1 & 3 \\ 0 & 2\end{array}\right)$ has two admissible arrangements: one of them consists of the pairs $(0,1)$ and $(2,3)$; the other one consists of the pairs $(1,2),(0,3)$. As another example,

$$
Z=\left(\begin{array}{lll}
1 & 3 & 5 \\
0 & 2 & 1
\end{array}\right)
$$

has five admissible arrangements:

the first one consists of $(0,1),(2,3),(4,5)$,

the second one consists of $(1,2),(3,4),(0,5)$,

the third one consists of $(1,2),(4,5),(0,3)$, 


$$
\begin{aligned}
& \text { the fourth one consists of }(0,1),(3,4),(2,5) \text {, } \\
& \text { the fifth one consists of }(2,3),(1,4),(0,5) \text {. }
\end{aligned}
$$

Now let $\Phi$ be an admissible arrangement for $Z$. If $\Psi$ is any subset of $\Phi$, we denote by $\Psi^{*}$ (resp. $\Psi_{*}$ ) the set of singles in the first row (resp. second row) of $Z$ which appear in a pair of $\Psi$. Let $Z_{2}$ be the set of elements which appear in both rows of $Z$.

Let $\hat{\Phi}$ be a subset of $\Phi$. We define

$$
\underline{c}(Z, \Phi, \hat{\Phi})=\sum_{\Psi \subset \Phi}(-1)^{e(\Psi)}\left[\begin{array}{l}
Z_{2} \amalg \Psi_{*} \amalg(\Phi-\Psi)^{*} \\
Z_{2} \amalg \Psi^{*} \amalg(\Phi-\Psi)_{*}
\end{array}\right]
$$

where $e(\Psi)=\left|\hat{\Phi}^{*} \cap \Psi^{*}\right|$. This sum is interpreted as follows. If $|\hat{\Phi}|$ is even, then $(-1)^{e(\Psi)}=(-1)^{e(\Phi-\Psi)}$ and we take the sum over equivalence classes of subsets $\Psi \subset \Phi$ ( $\Psi$ is equivalent to $\Phi-\Psi$ ); the expression in square brackets is interpreted as an irreducible representation of $D_{n}$ (it is the same for $\Psi$ and for $\Phi-\Psi$ ). Thus the sum has $2^{d-1}$ terms; it is a virtual representation of $D_{n}$. If $|\hat{\Phi}|$ is odd, then $(-1)^{e(\Psi)}=-(-1)^{e(\Phi-\Psi)}$ and our sum with $2^{d}$ terms is an $t$-anti-invariant virtual representation of $\hat{D}_{n}$.

In any case $\underline{c}(Z, \Phi, \hat{\Phi})$ is said to be a (nondegenerate) virtual cell.

1.11. If we define $\bar{Z}$ (with $Z$ nondegenerate) as in 1.4 , with respect to an integer $t \geqslant z_{2 m}$, we get again a nondegenerate special symbol of rank $n$ and defect 0 . We have a $1-1$ correspondence $z \leftrightarrow t-z$ between the singles in $Z$ and the singles in $\bar{Z}$. Given $\hat{\Phi} \subset \Phi$ for $Z$ as in 1.10 ( $\Phi$ admissible), we can thus transport them to $\bar{Z}$ and we get an admissible arrangement $\bar{\Phi}$ for $\bar{Z}$ and a subset $\overline{\hat{\Phi}}$ of $\bar{\Phi}$. We have

$$
\underline{c}(Z, \Phi, \hat{\Phi}) \otimes \operatorname{sign}^{\prime}=\underline{c}(\bar{Z}, \bar{\Phi}, \overline{\hat{\Phi}})
$$

1.12. We now consider the subgroup $\varsigma_{r} \times \hat{D}_{s}$ of $\hat{D}_{n}$ and the corresponding subgroup $\mathfrak{S}_{r} \times D_{s}$ of $D_{n}(r+s=n, r \geqslant 1, s \geqslant 1)$. Let

$$
Z^{\prime}=\left(\begin{array}{c}
z_{2}^{\prime}, z_{4}^{\prime}, \ldots, z_{2 m}^{\prime} \\
z_{1}^{\prime}, z_{3}^{\prime}, \ldots, z_{2 m-1}^{\prime}
\end{array}\right)
$$

be a nondegenerate special symbol of rank $s$ and defect 0 ; we shall assume, as we may, that $2 m \geqslant r$. We associate to $Z^{\prime}$ the nondegenerate special symbol of rank $n$ and defect 0

$$
Z=\left(\begin{array}{c}
z_{2}, z_{4}, \ldots, z_{2 m} \\
z_{1}, z_{3}, \ldots, z_{2 m-1}
\end{array}\right)
$$

defined by $z_{i}=z_{i}^{\prime}(1 \leqslant i \leqslant 2 m-r), z_{i}=z_{i}^{\prime}+1(2 m+1-r \leqslant i \leqslant 2 m)$. Suppose that we are given an admissible arrangement $\Phi^{\prime}$ for $Z^{\prime}$ and a subset $\hat{\Phi}^{\prime}$ of $\Phi^{\prime}$. We transport these to $Z$ using the natural bijection $z_{i}^{\prime} \leftrightarrow z_{i}$ between $Z^{\prime}$ and $Z$. In the case where $r=2 m$ or $r \leqslant 2 m-1$ and $z_{2 m-1-r}^{\prime}<z_{2 m-r}^{\prime}$ (so that $Z, Z^{\prime}$ have the same number of singles) we thus get an admissible arrangement $\Phi$ for $Z$ and a subset $\hat{\Phi}$ of $\Phi$. In the case where $z_{2 m-1-r}^{\prime}=z_{2 m-r}^{\prime}$ (so that $Z$ has two new singles in addition to those coming from $Z^{\prime}$ ), the set of pairs in $Z$ coming from those in $\Phi^{\prime}$ together with the new pair $\left(z_{2 m-1-r}, z_{2 m-r}\right)$ form an admissible arrangement $\Phi$ for $Z$. It has a 
subset $\hat{\Phi}$ corresponding to the pairs in $\hat{\Phi}^{\prime}$ (the new pair is not in $\hat{\Phi}$ ). Using now the results in 1.7 we see that

$$
J_{\Phi_{r}^{n} \times D_{s}}^{D_{n}}\left(\varepsilon(r) \otimes \underline{c}\left(Z^{\prime}, \Phi^{\prime}, \hat{\Phi}^{\prime}\right)\right)=\underline{c}(Z, \Phi, \hat{\Phi})
$$

if $\left|\hat{\Phi}^{\prime}\right|$ is even and that the same formula holds when $\left|\hat{\Phi}^{\prime}\right|$ is odd, provided that we replace $D_{s}, D_{n}$ by $\hat{D}_{s}, \hat{D}_{n}$.

1.13. Let $Z, \Phi, \hat{\Phi}$ be as in 1.11. Let $\Phi_{1}$ be the set of pairs $\left(z_{i}, z_{j}\right)$ in $\Phi$ such that $z_{i}+z_{j}$ is odd. Let $\Phi_{2} \subset \Phi$ be defined by $\Phi_{2}=\left(\hat{\Phi} \cup \Phi_{1}\right)-\left(\hat{\Phi} \cap \Phi_{1}\right)$. We have

$$
(-1)^{a[Z]} \zeta(\underline{c}(Z, \Phi, \hat{\Phi}))=\underline{c}\left(Z, \Phi, \Phi_{2}\right)
$$

where $\zeta$ is defined in 1.5 . The proof is the same as that of $[4,2.18]$.

1.14. Now let

$$
Z=\left(\begin{array}{l}
z_{1}, z_{3}, z_{5}, \ldots, z_{2 m-1} \\
z_{1}, z_{3}, z_{5}, \ldots, z_{2 m-1}
\end{array}\right)
$$

be a degenerate special symbol of rank $n$ and defect 0 ( $n$ is necessarily even). It gives rise to two irreducible representations $[Z]_{b},[Z]_{b^{\prime}}$ of $D_{n}$ (see 1.7). They are said to be degenerate virtual cells. The tensor product of $[Z]_{b}$ or $[Z]_{b^{\prime}}$ with sign' $\mid D_{n}$ is $[\bar{Z}]_{b}$ or $[\bar{Z}]_{b^{\prime}}$ (not necessarily in this order), where $\bar{Z}$ is defined as in 1.4 , with respect to an integer $t \geqslant z_{2 m-1}$.

The behaviour of $[Z]_{b}$ under the operation $J$ is described in 1.7. We have

$$
\zeta\left([Z]_{b}\right)=(-1)^{i}[Z]_{b}, \quad \zeta\left([Z]_{b^{\prime}}\right)=(-1)^{i}\left[Z_{b^{\prime}}\right]
$$

where $i=a\left([Z]_{b}\right)=a\left([Z]_{b^{\prime}}\right)$.

1.15. We now define, by induction on $n \geqslant 1$, a certain set of involutions $\hat{\Omega}_{n} \subset \hat{D}_{n}$. For $n=1$, we take $\hat{\Omega}_{1}=\hat{D}_{1}$. Assume now that $n \geqslant 2$ and that $\hat{\Omega}_{s} \subset \hat{D}_{s}$ is already defined for $1 \leqslant s<n$. We say that $w \in \hat{D}_{n}$ is in $\hat{\Omega}_{n}$ if

(a) there exist a partition $n=r+s(s \geqslant 1, r \geqslant 1)$ and an element $z \in \hat{\Omega}_{s} \subset \hat{D}_{s}$ such that $w=w_{0}^{(r)} \cdot z \in \mathfrak{S}_{r} \times \hat{D}_{s} \subset \hat{D}_{n}$, or $w=\left(w_{0} \varphi^{n}\right)\left(w_{0}^{(r)} \cdot z\right)$, or

(b) $w$ is the longest element in $\Im_{n}^{(b)} \subset D_{n}$ or in $\Im_{n}^{\left(b^{\prime}\right)} \subset D_{n}$ (see 1.7), or

(c) $\left(w_{0} \varphi^{n}\right) w$ is the longest element in $\mathfrak{S}_{n}^{(b)}$ or in $\mathfrak{S}_{n}^{\left(b^{\prime}\right)}$. (Here $w_{0}$ is the longest element in $D_{n}$ and $w_{0}^{(r)}$ is the longest element in $\subseteq_{r}$.)

Using the results in 1.5-1.15 just as in [4, 2.20], one proves the following two results. (Although Propositions 1.16, 1.17 are stated separately, their proofs must be carried out simultaneously.)

Proposition 1.16. Assume that $n \geqslant 1$. The following 3 sets of virtual representations of $D_{n}$ coincide:

(a) $\left\{\alpha_{w} \mid w \in \hat{\Omega}_{n} \cap D_{n}\right\}$,

(b) $\left\{Q_{w} \mid w \in \hat{\Omega}_{n} \cap D_{n}\right\}$,

(c) the set of virtual cells $\underline{c}(Z, \Phi, \hat{\Phi})$ with $Z$ special nondegenerate of rank $n$, defect $0,|\hat{\Phi}|$ even, and the virtual cells $[Z]_{b},[Z]_{b^{\prime}}$ with $Z$ special degenerate of rank $n$, defect 0 . (The latter exist only for even $n$.) Moreover, if $\underline{c}(Z, \Phi, \hat{\Phi})$ or $[Z]_{b}$ or $[Z]_{b^{\prime}}$ is equal to $Q_{w}=\alpha_{w^{\prime}}\left(w, w^{\prime} \in \hat{\Omega}_{n} \cap D_{n}\right)$ then

$$
a[Z] \equiv l\left(w^{\prime}\right) \quad(\bmod 2), \quad \nu-A[Z] \equiv l(w) \quad(\bmod 2) \text {. }
$$


Proposition 1.17. Assume that $n \geqslant 1$. The following 3 sets of t-anti-invariant virtual representations of $\hat{D}_{n}$ coincide:

(a) $\left\{\alpha_{w} \mid w \in \hat{\Omega}_{n}, w \notin D_{n}\right\}$,

(b) $\left\{Q_{w} \mid w \in \hat{\Omega}_{n}, w \notin D_{n}\right\}$,

(c) the set of virtual cells $\underline{c}(Z, \Phi, \hat{\Phi})$ with $Z$ special nondegenerate of rank $n$, defect 0 , and with $|\hat{\boldsymbol{\Phi}}|$ odd. Moreover, if $\underline{c}(Z, \Phi, \hat{\Phi})=\mathbb{Q}_{w}=\alpha_{w^{\prime}}\left(w, w^{\prime} \in \hat{\Omega}_{n}, w, w^{\prime} \notin D_{n}\right)$ then

$$
a[Z] \equiv l\left(w^{\prime} \varphi\right) \quad(\bmod 2), \quad \nu-A[Z] \equiv l(w \varphi) \quad(\bmod 2) .
$$

Just as in [4, 2.21] we obtain from 1.16, 1.17 the following

Corollary 1.18. Assume that $n \geqslant 1$. Let $\underline{c}$ be a virtual cell as in 1.16(c) and let $w \in D_{n}$ be such that $\underline{c}=\mathbb{Q}_{w}$. Then

$$
\underline{c}=\sum \operatorname{Tr}\left(T_{w}, \tilde{E} ;(l(w)-A(E)+\nu) / 2\right) E
$$

(sum over all irreducible $D_{n}$-modules $E$ ). If $\underline{c}$ is a virtual cell as in $1.17(\mathrm{c})$ and $w \in D_{n}$ is such that $\underline{c}=\mathbb{Q}_{w \varphi}$, then

$$
\underline{c}=\sum \operatorname{Tr}\left(T_{w} T_{\varphi}, \tilde{E} ;(l(w)-A(E)+\nu) / 2\right) E
$$

(sum over all irreducible nondegenerate $\hat{D}_{n}$-modules $E$ ). In both cases, all irreducible components $E$ of $\underline{c}$ have the same $a(E)$ and the same $A(E)$. The common value of $a(E)$ is called $a(\underline{c})$ and the common value of $A(E)$ is called $A(\underline{c})$.

LEMMA 1.19. Let $E$ be an irreducible, nondegenerate representation of $\hat{D}_{n}$. There exist a nondegenerate special symbol $Z$ of rank $n$ and defect 0 , an admissible arrangement $\Phi$ for $Z$ and a subset $\Psi \subset \Phi$ such that with the notation of 1.10 , we have

$$
E=\left[\begin{array}{c}
Z_{2} \amalg \Psi_{*} \amalg(\Phi-\Psi)^{*} \\
Z_{2} \amalg \Psi^{*} \amalg(\Phi-\Psi)_{*}
\end{array}\right] .
$$

We have

$$
\begin{gathered}
E \mid D_{n}=2^{-(d-1)} \sum_{\substack{\hat{\Phi} \subset \Phi \\
|\hat{\Phi}|=\text { even } .}}(-1)^{e^{\prime}(\hat{\Phi})} \underline{c}(Z, \Phi, \hat{\Phi}), \\
E-\iota(E)=2^{-(d-1)} \sum_{\substack{\hat{\Phi} \subset \Phi \\
|\hat{\Phi}|=\text { odd }}}(-1)^{e^{\prime}(\hat{\Phi})} \underline{c}(Z, \Phi, \hat{\Phi}),
\end{gathered}
$$

where $2 d$ is the number of singles in $Z$ and

$$
e^{\prime}(\hat{\Phi})=\left|\hat{\Phi}^{*} \cap \Psi^{*}\right|
$$

The proof is the same as that of Lemma 2.22 in [4].

\section{A combinatorial result.}

2.1. Let $Y$ be a vector space of dimension $2 d-1(d \geqslant 1)$ over the field $F_{2}$, endowed with a basis $e_{1}, e_{2}, \ldots, e_{2 d-1}$ and with a symplectic form ( , ): $Y \times Y \rightarrow F_{2}$ 
such that

$$
\left(e_{i}, e_{j}\right)= \begin{cases}1 & \text { if }|i-j|=1 \\ 0 & \text { otherwise }\end{cases}
$$

This form is singular; its radical is spanned by the vector $\varepsilon=e_{1}+e_{3}+e_{5}$ $+\cdots+e_{2 d-1}$.

If $d \geqslant 2$, we consider for each $i, 1 \leqslant i \leqslant 2 d-1$, the vector space

$$
Y_{i}=\left\{y \in Y \mid\left(e_{i}, y\right)=0\right\} /\left\{0, e_{i}\right\} .
$$

It inherits from $Y$ a symplectic form and a basis:

$$
\begin{array}{ll}
e_{1}, e_{2}, \ldots, e_{i-2}, e_{i-1}+e_{i+1}, e_{i+2}, \ldots, e_{2 d-1} & \text { if } i \neq 1,2 d-1, \\
e_{3}, e_{4}, \ldots, e_{2 d-1} & \text { if } i=1, \\
e_{1}, e_{2}, \ldots, e_{2 d-3} & \text { if } i=2 d-1 .
\end{array}
$$

This basis has the same relation to the symplectic form on $Y_{i}$ as the basis $e_{1}, \ldots, e_{2 d-1}$ had with (, ) on $Y$.

2.2. We define by induction on $d$, a family $\mathscr{F}(Y)$ of maximal isotropic subspaces of $V$, depending on the given basis $\left(e_{i}\right)$ of $Y$. If $d=1, \mathscr{F}(Y)$ consists of $Y$. Assume now that $d \geqslant 2$ and that $\mathscr{F}\left(Y_{i}\right)$ has been already defined for $1 \leqslant i \leqslant 2 d-1$ (with respect to the basis of $Y_{i}$ described above). By definition, a $d$-dimensional subspace $C$ of $Y$ is in $\mathscr{F}(Y)$ if and only if there exists $i, 1 \leqslant i \leqslant 2 d-1$, such that $e_{i} \in C$ and such that the image of $C$ under the natural map $\left\{y \in Y \mid\left(e_{i}, y\right)=0\right\} \rightarrow Y_{i}$ is in $\mathscr{F}\left(Y_{i}\right)$. For example, if $d=2, \mathscr{F}(Y)$ consists of two subspaces: the first is spanned by $e_{1}, e_{3}$ and the second is spanned by $e_{2}, e_{1}+e_{3}$. If $d=3, \mathscr{F}(Y)$ consists of five subspaces: the first one is spanned by $e_{1}, e_{3}, e_{5}$, the second one is spanned by $e_{1}, e_{4}, e_{3}+e_{5}$, the third is spanned by $e_{1}+e_{3}, e_{2}, e_{5}$, the fourth one is spanned by $e_{2}, e_{4}, e_{1}+e_{3}+e_{5}$, and the fifth is spanned by $e_{3}, e_{2}+e_{4}, e_{1}+e_{5}$.

The following properties of a subspace $C \in \mathscr{F}(Y)$ follow easily from the definitions.

(2.2.1) If $e_{i} \in C$, then the image of $C$ in $Y_{i}$ is in $\mathscr{F}\left(Y_{i}\right)$.

(2.2.2) If $e_{1} \in C$ and $d \geqslant 2$, then some $e_{i}, i \geqslant 2$, is in $C$.

(2.2.3) If $e_{2 d-1} \in C$ and $d \geqslant 2$, then some $e_{i}, i \leqslant 2 d-2$, is in $C$.

Let $\tilde{Y}$ be the set of vectors in $Y$ which lie in some subspace in $\mathscr{F}\left(Y_{i}\right)$. We have

Lemma 2.3. Assume that $d \geqslant 2$. Given $i, 1 \leqslant i \leqslant 2 d-1$, and two elements $y, y^{\prime} \in \tilde{Y}$ such that $\left(y, e_{i}\right)=\left(y^{\prime}, e_{i}\right)=1$, there exist a sequence of elements $y=y_{1}, y_{2}, \ldots, y_{m}=$ $y^{\prime}$ in $\tilde{Y}$ and a sequence of subspaces $C_{1}, C_{2}, \ldots, C_{m-1}$ in $\mathcal{F}(Y)$ such that $y_{h}, y_{h+1} \in C_{h}$ $(1 \leqslant h \leqslant m-1)$ and $\left(y_{h}, e_{i}\right)=1(1 \leqslant h \leqslant m)$.

Proof. Assume first that $i \neq 2 d-1$. Consider the vector space $\bar{Y}=Y /\{0, \varepsilon\}$ with the basis $\bar{e}_{1}, \bar{e}_{2}, \ldots, \bar{e}_{2 d-2}$ (images of $e_{1}, e_{2}, \ldots, e_{2 d-2}$ ) and with the nonsingular symplectic form induced by (, ). This is a vector space with a basis and form of the kind considered in $[4,3.1]$. In particular, we can define a family of lagrangian subspaces $\mathscr{F}(\bar{Y})$ of $\bar{Y}$ as in [4, 3.2]. It is clear that the inverse images under $Y \rightarrow \bar{Y}$ of the subspaces in $\mathscr{F}(\bar{Y})$ are precisely the subspaces in $\mathscr{F}(Y)$ which contain a basis element $e_{i}(i \leqslant 2 d-2)$. By (2.2.3) all subspaces in $\mathscr{F}(Y)$ are of this type. Therefore, in this case, our lemma follows from [4, Lemma 3.7]. 
The case where $i \neq 1$ is entirely similar: one considers the basis $\bar{e}_{2}, \ldots, \bar{e}_{2 d-2}, \bar{e}_{2 d-1}$ of $\bar{Y}$.

Since $d \geqslant 2$, one of the inequalities $i \neq 1, i \neq 2 d-1$ must be satisfied and the lemma is proved.

2.4. Now let $X$ be another vector space of dimension $2 d-1$, and let $\langle$,$\rangle :$ $X \times Y \rightarrow F_{2}$ be a nonsingular bilinear pairing. We denote by $e_{i}^{\prime}$ the vector in $X$ defined by $\left\langle e_{i}^{\prime}, y\right\rangle=\left(e_{i}, y\right), y \in Y, 1 \leqslant i \leqslant 2 d-1$.

We have

Proposition 2.5. Let $x, y \rightarrow[x, y]$ be a map $X \times \tilde{Y} \rightarrow F_{2}$ with the following properties.

(a) For any $x \in X$ and any $C \in \mathscr{F}(Y)$, the function $y \rightarrow[x, y]\left(C \rightarrow F_{2}\right)$ is $F_{2}$-linear.

(b) For any $x \in X, y \in \tilde{Y}$ and $e_{j}$ such that $\left\langle x, e_{j}\right\rangle=0$, we have

$$
(-1)^{[x, y]}+(-1)^{\left[x+e_{j}^{\prime}, y\right]}=(-1)^{\langle x, y\rangle}+(-1)^{\left\langle x+e_{j}^{\prime}, y\right\rangle} .
$$

(c) For any $y \in \tilde{Y}$, we have

$$
\sum_{x \in X}(-1)^{[x, y]}=\sum_{x \in X}(-1)^{\langle x, y\rangle}
$$

Then $[x, y]=\langle x, y\rangle$ for all $x \in X, y \in \tilde{Y}$.

The proof is almost identical to that of Proposition 3.8 in [4]. It uses Lemma 2.3 instead of $[4,3.7]$.

REMARK 2.6. We shall later apply this result in the following case. We take $Y$ to be the set of all subsets of even cardinality of $Z_{1}=\{0,1,2, \ldots, 2 d-1\}$. It is an $F_{2}$-vector space with respect to the addition defined in $I, I^{\prime} \rightarrow\left(I \cup I^{\prime}\right)-\left(I \cap I^{\prime}\right)$. The subsets $e_{i}=\{i-1, i\}(1 \leqslant i \leqslant 2 d-1)$ form a basis for $Y$. The symplectic form on $Y$ is $I, I^{\prime} \rightarrow\left|I \cap I^{\prime}\right| \bmod 2$. If $I \in Y$, then the condition that $I \in \tilde{Y}$ is that $|I \cap\{0,2,4, \ldots\}|=|I \cap\{1,3,5, \ldots\}|$.

We take $X$ to be the set of all subsets of $Z_{1}=\{0,1, \ldots, 2 d-1\}$ (with the $F_{2}$-vector space structure defined just as for $Y$ ) taken modulo the subspace spanned by $Z_{1}$ itself. (Thus an element of $X$ is an unordered pair $\left(I, Z_{1}-I\right)$, where $I \subset Z_{1}$.) The duality pairing $\langle\rangle:, X \times Y \rightarrow F_{2}$ is defined by $\langle I, J\rangle=|I \cap J| \bmod 2$. (Note that $\left|\left(Z_{1}-I\right) \cap J\right| \equiv|I \cap J| \bmod 2$ whenever $\left.|J| \equiv 0 \bmod 2.\right)$

The element $e_{i}^{\prime} \in X$ is the set $\{i-1, i\}$ together with its complement in $Z_{1}$.

\section{The main results.}

3.1. Let $k$ be an algebraic closure of the finite field $F_{q}$ with $q$ elements. Let $V$ be a $k$-vector space of dimension $2 n(n \geqslant 1)$ endowed with a nonsingular quadratic form. Let $G$ be the full orthogonal group of this quadratic form; it has two connected components. The identity component is denoted $G^{0}$. Let $e_{1}, f_{1}, e_{2}, f_{2}, \ldots, e_{n}, f_{n}$ be a basis of $V$ with the following properties: the inner product of $e_{i}$ with $f_{i}$ is 1 $(1 \leqslant i \leqslant n)$, all other inner products of basis elements are zero and the quadratic form has value zero at all basis elements. Let $F: V \rightarrow V$ be the Frobenius semilinear map for an $F_{q}$-rational structure on $V$ such that $F\left(e_{i}\right)=\left(e_{i}\right), F\left(f_{i}\right)=f_{i}(1 \leqslant i \leqslant n)$. We shall also denote by $F: G \rightarrow G$ the map satisfying $F(g e)=F(g) F(e)$ for all 
$g \in G, e \in V$. This is the Frobenius map for an $F_{q}$-rational structure on $G$. Its fixed point set $G^{F}$ is the full orthogonal group of an $F_{q}$-split quadratic form in $2 n$ variables. Let $j: V \rightarrow V$ be the linear map which interchanges $e_{1}$ and $f_{1}$ and leaves the other basis vectors unchanged. Then $j \in G-G^{0}, j^{2}=1$. Let $F^{\prime}=j F=F j: V \rightarrow V$. We shall also denote by $F^{\prime}: G \rightarrow G$ the map satisfying $F^{\prime}(g e)=F^{\prime}(g) F^{\prime}(e)$ for all $g \in G, e \in V$; thus, $F^{\prime}(g)=j F^{\prime}(g) j(g \in G)$. This is the Frobenius map for an $F_{q}$-rational structure on $G$ such that the fixed point set $G^{F^{\prime}}$ is the full orthogonal group of a nonsplit quadratic form over $F_{q}$ in $2 n$ variables.

3.2. Let $\mathscr{B}$ be the variety of all Borel subgroups of $G$. Then $F: \mathscr{B} \rightarrow \mathscr{B}, F^{\prime}: \mathscr{B} \rightarrow \mathscr{B}$ define two $F_{q}$-rational structures on $\mathscr{B}$. We identify the Weyl group $W$ of $G^{0}$ with the Coxeter group $D_{n}$; when $n \geqslant 2$, the simple reflections $b, b^{\prime}$ of $D_{n}$ correspond to the two families of maximal isotropic subspaces in $V$. Thus, the orbits of $G^{0}$ on $\mathscr{B} \times \mathscr{B}_{B}$ are parametrized by the elements of $D_{n}$. Given $w \in D_{n}$, we denote by $\mathscr{B}_{w}$ the variety of all $B \in \mathscr{B}$ such that $B, F B$ are in relative position $w$; the variety $\mathscr{B}_{w}^{\prime}$ is defined in the same way, using $F^{\prime}$ instead of $F$. The finite group $\left(G^{0}\right)^{F}$ (resp. $\left.\left(G^{0}\right)^{F^{\prime}}\right)$ acts naturally, by conjugation, on $\mathscr{B}_{w}$ (resp. on $\mathfrak{B}_{w}^{\prime}$ ), and we thus have virtual representations

$$
R_{w}=\sum_{i}(-1)^{i} H_{c}^{i}\left(\mathscr{B}_{w}, \mathbf{Q}_{l}\right) \text { of }\left(G^{0}\right)^{F}
$$

and

$$
R_{w}^{\prime}=\sum_{i}(-1)^{i} H_{c}^{i}\left(\mathscr{B}_{w}^{\prime}, \mathbf{Q}_{l}\right) \text { of }\left(G^{0}\right)^{F^{\prime}}
$$

For each $M \in \Re\left(D_{n}\right)$, we define

$$
R(M)=\left|D_{n}\right|^{-1} \sum_{w \in D_{n}} \operatorname{Tr}(w, M) R_{w} \in \Re\left(\left(G^{0}\right)^{F}\right) \otimes \mathbf{Q}
$$

(the Grothendieck group of virtual $\overline{\mathbf{Q}}_{\Gamma}$ representations of $\left(G^{0}\right)^{F}$, tensored with $\mathbf{Q}$ ). For each $M \in \Re\left(\hat{D}_{n}\right)^{-}$, we define

$$
R(M)=\left|\hat{D}_{n}\right|^{-1} \sum_{w \in D_{n}} \operatorname{Tr}(w \varphi, M) R_{w}^{\prime} \in \Re\left(\left(G^{0}\right)^{F^{\prime}}\right) \otimes \mathbf{Q}
$$

We have

Proposition 3.3. Let $\underline{c} \in \Re\left(D_{n}\right)$ be a virtual cell and let $a=a(\underline{c})$ (see 1.18). Then $R(c)$ is a linear combination with integral positive coefficients of irreducible representations of $\left(G^{0}\right)^{F}$ plus a linear combination with integral coefficients of elements $R(E)$ where $E$ runs through the irreducible $Q\left[D_{n}\right]$-modules such that $a(E)>a$. (For such $E$, we have $\operatorname{dim} R(E) \equiv 0\left(\bmod q^{a}\right)$.)

The proof is the same as that of Corollary 4.9 in [4], using 1.18 instead of [4, 2.20]. As in that proof, we must make use of the fact that $\left(G^{0}\right)^{F}$ can have unipotent cuspidal representations only for even values of $n$ (cf. [2]).

3.4. We shall also need an analogue of Proposition 3.3 for the case of the twisted orthogonal group. This case is not covered by the arguments in Asai's paper [1]. We shall, instead, use Theorem 3.8 of [6] to deduce the following theorem. 
THEOREM 3.5.

$$
\sum(-1)^{i} H_{c}^{i}\left(\mathscr{B}_{w}^{\prime}, \mathbf{Q}_{l}\right)^{(h)}=\frac{1}{2} \sum_{E} \operatorname{Tr}\left(T_{w} T_{\varphi}, \tilde{E} ; h / 2\right) R(E-\iota(E))
$$

(sum over all irreducible, nondegenerate representations $E$ of $\hat{D}_{n}$ ), as elements of $\Re\left(\left(G^{0}\right)^{F^{\prime}}\right) \otimes \mathbf{Q}$. (The superscript ${ }^{(h)}$ denotes the part of weight $\left.h.\right)$

Just as in $[4,4.8]$ the previous theorem, together with $[4,4.5]$, shows that, for any $w \in D_{n}$ and any integer $h$, the element of $\Re\left(\left(G^{0}\right)^{F^{\prime}}\right) \otimes \mathbf{Q}$ given by

$$
\frac{(-1)^{h}}{2} \sum_{\substack{E \\ \text { nondeg. }}} \sum_{y \leqslant w} \sum_{j \geqslant 0} P_{y, w, j} \operatorname{Tr}\left(T_{y} T_{\varphi}, \tilde{E} ; \frac{h}{2}-j\right) R(E-\iota(E))
$$

is a linear combination with integral positive coefficients of irreducible representations of $\left(G^{0}\right)^{F^{\prime}}$. Here $P_{y, w, j}$ are certain integers defined in [4, 4.4]. From this, and from 1.18 , just as in $[4,4.9]$ we deduce

Proposition 3.6. Let $\underline{c} \in \mathscr{R}\left(\hat{D}_{n}\right)^{-}$be a virtual cell and let $a=a(\underline{c})$ (see 1.18). Then $R(\underline{c})$ is a linear combination with integral positive coefficients of irreducible representations of $\left(G^{0}\right)^{F^{\prime}}$ plus a linear combination with integral coefficients of elements $R(E-\iota(E))$ where $E$ runs through the irreducible nondegenerate $\mathbf{Q}\left[\hat{D}_{n}\right]$-modules such that $a(E)>a$. (For such $E$, we have $\operatorname{dim} R(E-\iota(E)) \equiv 0\left(\bmod q^{a}\right)$.)

As in the proof of Corollary 4.9 in [4] we must make use of the fact that $\left(G^{0}\right)^{F^{\prime}}$ can have unipotent cuspidal representations only in the case where the semisimple $F_{q}$-rank of $\left(G^{0}\right)^{F^{\prime}}$ is even, i.e., $n$ is odd (cf. [2]).

3.7. For each integer $1 \leqslant r \leqslant n-1$ we denote by $P_{r, s}(s=n-r)$ the stabilizer in $G^{0}$ of the isotropic subspace spanned by $e_{n}, e_{n-1}, \ldots, e_{n-r+1}$. It is a parabolic subgroup of $G^{0}$, stable under $F$ and $F^{\prime}$. It has as Levi subgroup the group $L_{r, s}$ stabilizing the subspace spanned by $e_{n}, e_{n-1}, \ldots, e_{n-r+1}$ and the subspace spanned by $f_{n}, f_{n-1}, \ldots, f_{n-r+1}$. Note that $L_{r, s}$ is again stable under $F$ and $F^{\prime}$ and it is isomorphic to the product of $G L_{r}$ with a special orthogonal group in $2 s$ variables which is split with respect to $F$ and nonsplit with respect to $F^{\prime}$.

Assume that notations have been chosen such that under the 1-1 correspondence between classes of maximal parabolics in $G^{0}$ and simple reflections in the Weyl group $D_{n}$ of $G^{0}$ the stabilizer $P_{b}$ of the subspace spanned by $e_{n}, e_{n-1}, \ldots, e_{2}, e_{1}$ corresponds to $b \in D_{n}$, while the stabilizer $P_{b^{\prime}}$ of the subspace spanned by $e_{n}, e_{n-1}, \ldots, e_{2}, f_{1}$ corresponds to $b^{\prime} \in D_{n}$. Then $P_{b}, P_{b^{\prime}}$ are $F$-stable (but not $F^{\prime}$ stable) and admit $F$-stable Levi subgroups isomorphic to $G L_{n}$.

The unipotent representations of $\left(G^{0}\right)^{F}$ and $\left(G^{0}\right)^{F^{\prime}}$ have been classified in [2] in terms of symbols of rank $n$ and even defect.

Recall that a symbol of rank $n$ and even defect is an unordered pair $\Lambda=\left(\begin{array}{l}T^{\prime \prime} \\ T^{\prime \prime}\end{array}\right)$ consisting of two finite subsets $T^{\prime}, T^{\prime \prime}$ of $\{0,1,2,3, \ldots\}$ such that $\left|T^{\prime}\right|+\left|T^{\prime \prime}\right|=2 \mathrm{~m}$, $\Sigma_{\lambda \in T^{\prime}} \lambda+\Sigma_{\mu \in T^{\prime \prime}} \mu=n+m^{2}-m$. (The defect of $\Lambda$ is ||$T^{\prime}|-| T^{\prime \prime}||$.)

There is an equivalence relation on such pairs generated by the shift 


$$
\left(\begin{array}{c}
T^{\prime} \\
T^{\prime \prime}
\end{array}\right) \sim\left(\begin{array}{c}
0 \amalg\left(T^{\prime}+1\right) \\
0 \amalg\left(T^{\prime \prime}+1\right)
\end{array}\right)
$$

and we shall often identify a symbol with its equivalence class. This notion contains as a special case the notion of special symbol of rank $n$ and defect 0 considered in 1.9. A symbol such that $T^{\prime} \neq T^{\prime \prime}$ is said to be nondegenerate. A symbol such that $T^{\prime}=T^{\prime \prime}$ is said to be degenerate; it is then automatically special. Any symbol $\Lambda$ of rank $n$ and even defect gives rise to a special symbol $Z$ of rank $n$ and defect 0 , as follows. We take the entries of $\Lambda$ and arrange them in a single row in increasing order. We get a monotonic sequence in which there may be equalities but no two consecutive equalities. The first, third, fifth, etc., term of this sequence will be the second row of $Z$ while the second, fourth, etc., term of this will be the first row of $Z$. Thus $Z$ is the unique special symbol of defect 0 whose set of entries (some of which may be repeated twice) coincides with the set of entries of $\Lambda$ (union of $T^{\prime}$ and $T^{\prime \prime}$, with common elements repeated twice). We shall then set $a_{\Lambda}=a([Z])$, if $Z$ is nondegenerate, $a_{\Lambda}=a\left([Z]_{b}\right)=a\left([Z]_{b^{\prime}}\right)$ if $Z$ is degenerate. In the case where $\Lambda$ has rank $n$ and defect 0 , we have $a_{\Lambda}=a[\Lambda]$ (see 1.6). The following lemma is a consequence of the results in $1.7,(1.4 .1),[4,2.4,2.7]$, and the results in [2] on classification and degrees of unipotent representations. (Compare Lemma 5.2 in [4].)

LEMMA 3.8. There exists a 1-1 correspondence $\Lambda \leftrightarrow \rho(\Lambda)$ between the set of symbols of rank $n$ and defect $\equiv 2(\bmod 4)($ up to shift $)$ and the set of unipotent representations ( up to isomorphism) of $\left(G^{0}\right)^{F^{\prime}}$, with the properties (i), (ii), (iii) below.

There exists a correspondence $\Lambda \leftrightarrow \rho(\Lambda)$ between the set of symbols of rank $n$ and defect $\equiv 0(\bmod 4)(u p$ to shift) and the set of unipotent representations (up to isomorphism) of $\left(G^{0}\right)^{F}$, which is 1-1, except that to each degenerate symbol $\Lambda$ (of defect $0)$ there correspond two unipotent representations $\rho(\Lambda)_{b}, \rho(\Lambda)_{b^{\prime}}$, and which has the properties (i), (ii), (iii) below.

(i) If $Z$ is the special symbol corresponding to $\Lambda$ (of rank $n$ and even defect), $a=a_{\Lambda}$ and $d=d[Z]$ is such that $2 d$ is the number of singles of $Z$, then

$2^{d-1} \operatorname{dim} \rho(\Lambda) \equiv q^{a}\left(\bmod q^{a+1}\right)$ if $\Lambda$ is nondegenerate, $\operatorname{dim} \rho(\Lambda)_{b}=\operatorname{dim} \rho(\Lambda)_{b^{\prime}} \equiv$ $q^{a}\left(\bmod q^{a+1}\right)$ if $\Lambda$ is degenerate.

(ii) Let $\Lambda$ be a symbol of rank $n$ and even defect. Let $t$ be an integer, $t \geqslant$ all entries in $\Lambda$. Let $\bar{T}^{\prime}=\left\{t-i \mid 0 \leqslant i \leqslant t, i \notin T^{\prime \prime}\right\}, \bar{T}^{\prime \prime}=\left\{t-i \mid 0 \leqslant i \leqslant t, i \notin T^{\prime}\right\}$, and let $\bar{\Lambda}=\left(\bar{T}^{\prime \prime}\right)$. This is again a symbol of rank $n$, even defect and

$$
\begin{aligned}
& D(\rho(\Lambda))=\rho(\bar{\Lambda}) \text { if } \Lambda \text { is nondegenerate, } \\
& \left.\begin{array}{l}
D\left(\rho(\Lambda)_{b}\right)=\rho(\bar{\Lambda})_{b} \text { or } \rho(\bar{\Lambda})_{b^{\prime}} \\
D\left(\rho(\Lambda)_{b^{\prime}}\right)=\rho(\bar{\Lambda})_{b} \text { or } \rho(\bar{\Lambda})_{b^{\prime}}
\end{array}\right\} \quad \text { if } \Lambda \text { is degenerate, }
\end{aligned}
$$

where $D$ is the duality operator defined as in [4, (4.6.5)] (but sum only over those $P$ which are defined over $F_{q}$ ).

(iii) Let $\Lambda^{\prime}$ be a symbol of rank $s$ and even defect $(1 \leqslant s \leqslant n-1)$ and let $r=n-s$; we may assume that $\Lambda^{\prime}$ has $\geqslant r$ entries. We associate to $\Lambda^{\prime}$ a symbol $\Lambda$ (or two symbols $\Lambda_{\mathrm{I}}, \Lambda_{\mathrm{II}}$ ) of rank $n$ and same defect as $\Lambda^{\prime}$, by increasing by 1 each of the r largest entries 
in $\Lambda^{\prime}$ (as in 1.7, where the case of symbols of defect 0 was considered). If $\Lambda^{\prime}$ is nondegenerate of defect $\equiv 0(\bmod 4)$ then

$$
\operatorname{Ind}_{P_{r, s}^{F}}^{\left(G^{0}\right)}\left(\operatorname{St}_{r} \otimes \rho\left(\Lambda^{\prime}\right)\right)=\left\{\begin{array}{l}
\rho(\Lambda)+\tau \text { or } \\
\rho\left(\Lambda_{\mathrm{I}}\right)+\rho\left(\Lambda_{\mathrm{II}}\right)+\tau ;
\end{array}\right.
$$

if $\Lambda^{\prime}$ is of defect $\equiv 2(\bmod 4)$ then

$$
\operatorname{Ind}_{P_{r, s}^{F^{\prime}}}^{\left(G^{0}\right)^{F^{\prime}}}\left(\mathrm{St}_{r} \otimes \rho\left(\Lambda^{\prime}\right)\right)=\left\{\begin{array}{l}
\rho(\Lambda)+\tau \text { or } \\
\rho\left(\Lambda_{\mathrm{I}}\right)+\rho\left(\Lambda_{\mathrm{II}}\right)+\tau ;
\end{array}\right.
$$

if $\Lambda$ is degenerate, and $r$ is odd, then

$$
\operatorname{Ind}_{P_{r, s}^{F}}^{\left(G^{0}\right)^{F}}\left(\mathrm{St}_{r} \otimes \rho\left(\Lambda^{\prime}\right)_{b}\right)=\operatorname{Ind}_{P_{r, s}^{F}}^{\left(G^{0}\right)^{F}}\left(\mathrm{St}_{r} \otimes \rho\left(\Lambda^{\prime}\right)\right)=\rho(\Lambda) ;
$$

if $\Lambda$ is degenerate and $r$ is even, then

$$
\operatorname{Ind}_{P_{r, s}^{F}}^{\left(G^{0}\right)^{F}}\left(\operatorname{St}_{r} \otimes \rho\left(\Lambda^{\prime}\right)_{b}\right)=\rho(\Lambda)_{b}
$$

and the same holds with $b$ replaced by $b^{\prime}$.

Here, $\tau$ is a $\mathbf{Z}$-linear combination of representations $\rho\left(\Lambda_{i}\right)$ such that $a_{\Lambda_{i}}>a_{\Lambda}$ (or $\left.a_{\Lambda_{i}}>a_{\Lambda_{\mathrm{I}}}=a_{\Lambda_{\mathrm{II}}}\right)$ and $\mathrm{St}_{r}$ is the Steinberg representation of $G L_{n}\left(F_{q}\right)$.

Moreover,

$$
\begin{aligned}
\operatorname{Ind}_{P_{b}^{F}}^{\left(G^{0}\right)^{F}}\left(\mathrm{St}_{n}\right) & =\rho\left(\begin{array}{l}
1,2,3, \ldots, \frac{n}{2} \\
1,2,3, \ldots, \frac{n}{2}
\end{array}\right)+\tau \text { if } n \text { is even, } \\
& =\rho\left(\begin{array}{l}
1,2,3, \ldots, \frac{n+1}{2} \\
0,2,3, \ldots, \frac{n+1}{2}
\end{array}\right)+\tau \text { if } n \text { is odd },
\end{aligned}
$$

and the same holds with $b$ replaced by $b^{\prime}$. Here $\tau$ is a Z-linear combination of representations $\rho\left(\Lambda_{i}\right)$ such that $a_{\Lambda_{i}}>\frac{1}{2} n(n-1)$.

REMARK 3.9. The unipotent representations of $\left(G^{0}\right)^{F}$ or $\left(G^{0}\right)^{F^{\prime}}$ of form $\rho(\Lambda), \Lambda$ nondegenerate, are said to be nondegenerate. The other unipotent representations (which can only exist for $\left(G^{0}\right)^{F}$ ) are said to be degenerate. We have

LEMMA 3.10. For any unipotent representation $\rho$ of $\left(G^{0}\right)^{F}$ or of $\left(G^{0}\right)^{F^{\prime}}$ there exist integers $d=d(\rho) \geqslant 0, a=a(\rho) \geqslant 0$ such that

$$
\begin{aligned}
& \left.\begin{array}{l}
1 \leqslant d^{2} \leqslant n \\
2^{d-1} \operatorname{dim}(\rho) \equiv q^{a}\left(\bmod q^{a+1}\right)
\end{array}\right\} \quad \text { if } \rho \text { is nondegenerate } \\
& \left.\begin{array}{ll}
d=0 & \\
& \operatorname{dim}(\rho) \equiv q^{a}\left(\bmod q^{a+1}\right)
\end{array}\right\} \quad \text { if } \rho \text { is degenerate. }
\end{aligned}
$$

Let $\tilde{\boldsymbol{\sigma}}(n)$ be the largest integer such that $\tilde{\sigma}(n)^{2} \leqslant n$. If $q>2^{\tilde{\sigma}(n)-1}$ then the conditions (3.10.1), (3.10.2), (3.10.3), (3.10.4) determine $d(\rho), a(\rho)$ uniquely.

The proof is the same as that of Lemma 5.3 in [4]. 
LEMMA 3.11. If $\underline{c}$ is a virtual cell in $\Re\left(D_{n}\right)$ or $\Re\left(D_{n}\right)^{-}$, then

$$
\operatorname{dim} R(\underline{c}) \equiv q^{a(\underline{c})} \quad\left(\bmod q^{a(\underline{c})+1}\right) .
$$

This follows from [2, (2.6.2)]. (Compare Lemma 5.4 in [4].)

LEMMA 3.12. (i) Let $Z$ be a nondegenerate symbol of rank $n$ and defect 0 , with $2 d$ singles, let $\Phi$ be an admissible arrangement for $Z$ and let $\hat{\Phi}, \hat{\Phi}^{\prime}$ be two subsets of $\Phi$ such that $|\hat{\Phi}| \equiv\left|\hat{\Phi}^{\prime}\right|(\bmod 2)$. Let $\underline{c}=\underline{c}(Z, \Phi, \Phi), \underline{c}^{\prime}=\underline{c}\left(Z, \Phi, \Phi^{\prime}\right)$. Then

$$
\left\langle R(\underline{c}), R\left(\underline{c}^{\prime}\right)\right\rangle= \begin{cases}2^{d-1}, & \text { if } \hat{\Phi}=\hat{\Phi}^{\prime}, \\ 0, & \text { if } \hat{\Phi}=\hat{\Phi}^{\prime}\end{cases}
$$

(inner product over $\left(G^{0}\right)^{F}$ if $|\hat{\boldsymbol{\Phi}}|$ is even and over $\left(G^{0}\right)^{F^{\prime}}$ if $|\hat{\boldsymbol{\Phi}}|$ is odd).

(ii) If $Z$ is a degenerate symbol of rank $n$ and defect 0 then

$$
\left\langle R\left([Z]_{b}\right), R\left([Z]_{b}\right)\right\rangle=\left\langle R\left([Z]_{b^{\prime}}\right), R\left([Z]_{b^{\prime}}\right)\right\rangle=1
$$

(inner product over $\left.\left(G^{0}\right)^{F}\right)$.

The proof is similar to that of Lemma 5.5 in [4].

Proposition 3.13. Let $\underline{c}$ be a virtual cell in $\Re\left(D_{n}\right)$ or $\Re\left(D_{n}\right)^{-}$and let $Z$ be the corresponding special symbol. Let $a=a(\underline{c})$ be defined as in 1.18 and let $d=d(\underline{c})$ be such that $2 d$ is the number of singles in $\underline{c}$. Assume that $q>2^{2 \tilde{\sigma}(n)-1}(\tilde{\sigma}(n)$ as in Lemma 3.10). If $Z$ is degenerate, then $R(\underline{c})$ is an irreducible unipotent representation $\rho$ of $\left(G^{0}\right)^{F}$ satisfying $a(\rho)=a, d(\rho)=d=0$. If $Z$ is nondegenerate, then

$$
R(c)=\sum_{i=1}^{2^{d-1}} \rho_{i}
$$

where $\rho_{i}\left(1 \leqslant i \leqslant 2^{d-1}\right)$ are distinct irreducible representations of $\left(G^{0}\right)^{F}$ (if $\underline{c} \in \mathcal{R}\left(D_{n}\right)$ ) or of $\left(G^{0}\right)^{F^{\prime}}$ (if $\left.\underline{c} \in \mathcal{R}\left(D_{n}\right)^{-}\right)$satisfying $a\left(\rho_{i}\right)=a, d\left(\rho_{i}\right)=d$.

The proof is similar to that of Theorem 5.6 in [4].

3.14. Let $Z$ be a tableau as in 1.9 , and let $Z_{1}$ be the set of singles of $Z$. We can write $Z_{1}=Z_{1}^{*} \mathrm{U}\left(Z_{1}\right)_{*}$ where $Z_{1}^{*}$ (resp. $\left.\left(Z_{1}\right)_{*}\right)$ is the set of entries of $Z_{1}$ appearing in the first row (resp. second row) of $Z$. Let $d=\left|Z_{1}^{*}\right|=\left|\left(Z_{1}\right)_{*}\right|$. Let $Z_{2}$ be the set of elements which appear in both rows of $Z$. Thus,

$$
Z=\left(\begin{array}{c}
Z_{2} \amalg Z_{1}^{*} \\
Z_{2} \amalg\left(Z_{1}\right)_{*}
\end{array}\right)
$$

THEOREM 3.15. Assume that $q>2^{2 \tilde{\sigma}(n)-1}(\tilde{\sigma}(n)$ as in Lemma 3.10).

(i) If $Z$ is degenerate then

$$
R[Z]_{b}=\rho(Z)_{b}, \quad \rho[Z]_{b^{\prime}}=\rho(Z)_{b^{\prime}} .
$$

(ii) Assume now that $Z$ is nondegenerate, so that $Z_{1}$ is nonempty. For each subset $M \subset Z_{1}$ we denote $M^{\#}=M \cup\left(Z_{1}\right)_{*}-\left(M \cap\left(Z_{1}\right)_{*}\right)$. If $\left|M^{\prime}\right| \equiv d(\bmod 2),|M|=d$, then: 


$$
\begin{aligned}
\left\langle\rho\left(\begin{array}{l}
Z_{2} \amalg\left(Z_{1}-M^{\prime}\right) \\
Z_{2} \amalg M^{\prime}
\end{array}\right), \quad R\left[\begin{array}{l}
Z_{2} \amalg\left(Z_{1}-M\right) \\
Z_{2} \amalg M
\end{array}\right]\right\rangle_{\left(G^{0}\right)^{F}} & \\
& =(-1)^{\left|M^{*} \cap M^{\prime *}\right|} 2^{-(d-1)} .
\end{aligned}
$$

If $\left|M^{\prime}\right| \not d(\bmod 2),|M|=d$, then:

$$
\begin{array}{r}
\left\langle\rho\left(\begin{array}{l}
Z_{2} \amalg\left(Z_{1}-M^{\prime}\right) \\
Z_{2} \amalg M^{\prime}
\end{array}\right), \quad R\left(\left[\begin{array}{l}
Z_{2} \amalg\left(Z_{1}-M\right) \\
Z_{2} \amalg M
\end{array}\right]-\left[\begin{array}{l}
Z_{2} \amalg M \\
Z_{2} \amalg\left(Z_{1}-M\right)
\end{array}\right]\right)\right\rangle_{\left(G^{0}\right)^{F^{\prime}}} \\
=(-1)^{\left|M^{\#} \cap M^{\prime *}\right|} 2^{-(d-1) .} .
\end{array}
$$

Corollary 3.16. (i) Let $w \in D_{n}$. With the assumptions of 3.15(i), we have

$$
\left\langle\rho(Z)_{b}, R_{w}\right\rangle_{\left(G^{0}\right)^{F}}=\operatorname{Tr}\left(w,[Z]_{b}\right) .
$$

The same holds with $b$ replaced by $b^{\prime}$.

(ii) We preserve the assumptions of 3.15(ii). Let $w \in D_{n}$. If $M^{\prime} \subset Z_{1},\left|M^{\prime}\right| \equiv d$ $(\bmod 2)$, then

$$
\begin{aligned}
\left\langle\rho\left(\begin{array}{l}
Z_{2} \amalg\left(Z_{1}-M^{\prime}\right) \\
Z_{2} \amalg M^{\prime}
\end{array}\right),\right. & \left.R_{w}\right\rangle_{\left(G^{0}\right)^{F}} \\
& =2^{-d} \sum_{\substack{M \subset Z_{1} \\
|M|=d}}(-1)^{\left|M^{*} \cap M^{\prime *}\right|} \operatorname{Tr}\left(w,\left[\begin{array}{l}
Z_{2} \amalg\left(Z_{1}-M\right) \\
Z_{2} \amalg M
\end{array}\right]\right) .
\end{aligned}
$$

If $M^{\prime} \subset Z_{1},\left|M^{\prime}\right| \not d(\bmod 2)$, then

$$
\begin{aligned}
\left\langle\rho\left(\begin{array}{l}
Z_{2} \amalg\left(Z_{1}-M^{\prime}\right) \\
Z_{2} \amalg M^{\prime}
\end{array}\right),\right. & \left.R_{w}^{\prime}\right)_{\left(G^{0}\right)^{F^{\prime}}} \\
& =2^{-d} \sum_{\substack{M \subset Z_{1} \\
|M|=d}}(-1)^{\left|M^{*} \cap M^{\prime *}\right|} \operatorname{Tr}\left(w \varphi,\left[\begin{array}{l}
Z_{2} \amalg\left(Z_{1}-M\right) \\
Z_{2} \amalg M
\end{array}\right]\right) .
\end{aligned}
$$

3.17. Proof of Theorem 3.15. As in the proof of Theorem 5.8 in [4], we may reduce the general case to the case where

$$
Z=Z_{1}=\left(\begin{array}{l}
1,3,5, \ldots, 2 d-1 \\
0,2,4, \ldots, 2 d-2
\end{array}\right), \quad d \geqslant 1 .
$$

See $[4,5.10 \mathrm{~A}), \mathrm{B})]$. In this special case, we see as in $[4,5.10 \mathrm{C})]$ that the left-hand side of (3.15.1) must be of the form $(-1)^{\left[M^{\prime *}, M^{\#}\right]} 2^{-(d-1)}$ and the left-hand side of (3.15.2) must be of the form $(-1)^{\left[M^{\prime \#}, M^{\#}\right]} 2^{-(d-1)}$, where $\left[M^{\prime \#}, M^{\#}\right]$ is a certain integer modulo 2. Let $X, Y, \tilde{Y}$ be defined as in 2.6. Then $M^{\prime \#}, M^{\#} \rightarrow\left[M^{\prime \#}, M^{\#}\right]$ is a map [, ]: $X \times \tilde{Y} \rightarrow F_{2}$. As in [4,5.10C)] we see that this map satisfies the assumptions of Proposition 2.5. It follows that $\left[M^{\prime \#}, M^{\#}\right] \equiv\left|M^{\prime \#} \cap M^{\#}\right| \bmod 2$ for all $M^{\prime \#} \in X, M^{\#} \in \tilde{Y}$, and the theorem is proved. 
3.18. For each unipotent representation $\rho$ of $\left(G^{0}\right)^{F}$ there is a well-defined sign $\lambda_{\rho}= \pm 1$ such that, whenever $\rho$ is contained in a generalized eigenspace of $F$ : $H_{c}^{i}\left(X_{w}\right) \rightarrow H_{c}^{i}\left(X_{w}\right)$, the corresponding eigenvalue of $F$ is of the form $\lambda_{\rho} \cdot q^{k}$, where $k$ is an integer. If $\rho$ is degenerate, then $\lambda_{\rho}=1$. If $\rho$ is nondegenerate, of form

$$
\rho=\rho\left(\begin{array}{l}
Z_{2} \amalg\left(Z_{1}-M^{\prime}\right) \\
Z_{2} \amalg M^{\prime}
\end{array}\right)
$$

(as in 3.14, 3.15(ii)), with $\left|Z_{1}\right|=2 d>0, M^{\prime} \subset Z_{1},\left|M^{\prime}\right| \equiv d(\bmod 2)$, then $\lambda_{\rho}=$ $(-1)^{\left(\left|M^{\prime}\right|-d\right) / 2}$. This is proved in the same way as Proposition 6.6 in [4].

3.19. It is very likely that 3.15 and 3.16 hold without restrictions on $q$. Indeed, $\mathrm{T}$. Asai has recently shown (Unipotent characters of $S p_{2 n}$ and $S O_{2 n+1}$ over $F_{q}$ with small $q$ ) that the main result of [4] holds also for small $q$. No doubt, the same method should apply in the case of even orthogonal groups as well.

\section{REFERENCES}

1. T. Asai, On the zeta functions of the varieties $X(w)$ of the split classical groups and the unitary groups, preprint.

2. G. Lusztig, Irreducible representations of finite classical groups, Invent. Math. 43 (1977), 125-175.

3. __ Representations of finite Chevalley groups, C.B.M.S. Regional Conf. Series in Math., No. 39, Amer. Math. Soc., Providence, R. I., 1978.

4. Unipotent characters of the symplectic and odd orthogonal groups over a finite field, Invent. Math. 64 (1981), 263-296.

5. _ A class of irreducible representations of a Weyl group. II, Proc. Kon. Nederl. Akad. A85 (1982),

6. G. Lusztig, Characters of reductive groups over a finite field, Preprint, I.H.E.S., Paris, 1982.

Department of Mathematics, Massachusetts institute of Technology, Cambridge, MasSACHUSETTS 02139 